\title{
Electrical Potential Distribution for Multiple Charged Surfaces under a General Boundary Condition
}

\author{
Jyh-PING Hsu ${ }^{1}$ AND Ming-Tsan Tseng \\ Department of Chemical Engineering, National Taiwan University, Taipei, Taiwan 10617, Republic of China
}

Received April 22, 1996; accepted June 25, 1996

\begin{abstract}
The electrical potential distribution of a system containing multiple charged surfaces with a general boundary condition is investigated theoretically. Here, a surface can assume a constant potential/charge density, or an arbitrary combination of the two, i.e., a mixed boundary condition; the latter is of particular significance in practice. Typical example includes surfaces containing various ionizable functional groups, charge-regulated surfaces, dynamic surface conditions, and patchwise charged surfaces. A systematic iterative method is proposed for the resolution of the linearized Poisson- Boltzmann equation governing the electrical potential distribution of the system under consideration. The sufficient and necessary condition under which the method proposed is applicable is discussed. Since the coefficients in the expression for the boundary condition at surface can be an arbitrary function, the present problem is a generalized $R$ obin problem. The conventional constant potential (Dirichlet) problem and constant surface charge (Neumann) problem can be recovered as special cases of the present model. A criterion is proposed to decide whether the separation distances between particles is appropriate for various approximate procedures, e.g., pairwise addition and linear superposition. We show that a system containing a large number of surfaces can be simulated by one which has relatively few surfaces. (1) 1996 A cademic Press, Inc.

Key Words: Poisson - Boltzmann equation, linearized; multiple surfaces, charged; boundary conditions, general, mixed; multipole expansion technique; semianalytical solution, iterative procedure.
\end{abstract}

\section{INTRODUCTION}

The electrical potential distribution of a charged system in an electrolyte solution is governed by the Poisson-Boltzmann equation (PBE). The solution to this equation provides necessary information for the estimation of the electrostatic interactions between two charged surfaces. One of the key factors affecting the degree of difficulty to the resolution of a PBE is the type of boundary condition assigned. Often, a charged surface is assumed to remain either at constant potential or at constant charge density. The former leads to a Dirichlet problem, and the latter to a Neumann problem.

\footnotetext{
${ }^{1}$ To whom correspondence should be addressed.
}

Although constant potential and constant charge density models are idealized descriptions, they are widely adopted for the purpose of a simpler mathematical treatment. In practice, various surface conditions can be assumed. These include, for example, charge-regulated surfaces (1-7) and surfaces of dynamic nature (8). In these cases, a mixed boundary-valued problem, i.e., a certain combination of the potential and charge density is specified at surface, needs to be solved. An important special case of this type of problem is that which involves a nonuniform boundary condition (912), such as patchwise distribution of surface charges (13). In general, since the orthogonal property of the eigenfunctions associated with the general solution of a PBE cannot be employed directly, solving a mixed boundary-valued problem is nontrivial.

Previous efforts were mainly based on the interactions of two charged surfaces. Glendinning and Russel (14), for example, examined the electrostatic interaction between two identical spheres under Debye-Huckle condition for the case of fixed surface potential/charge density. A multipole expansion technique was used. Ohshima considered the potential distribution for a system that comprises two dissimilar spheres both at constant surface potential $(15,16)$ and at constant charge density (17). A reflection (Schwartz's) method was adopted in these studies. A similar approach was used in the analysis of the interaction between a hard sphere and an ion-penetrable sphere (18). Carnie and Chan (2) studied the interaction between two identical spherical surfaces. The boundary conditions assumed include constant potential, constant charge density, and a linear combination of the two; a multipole expansion approach was used which was also applied to the case of constant potential/charge density $(3,14)$, and to the linearized charge-regulation model $(1,3)$. The problem of two planar surfaces under a linearized charge-regulation condition was discussed by Carnie and Chan (7). Prieve and Ruckenstein (5) analyzed the nonlinear PBE for the case of two charge-regulated planar surfaces. The governing equation was solved numerically. This approach was also adopted by Carnie et al. (6) and applied to the problem of two interacting spherical particles with a charge-regulated surface. 
In the present study we consider the solution to the linearized PBE under a general boundary condition. An arbitrary combination of potential and charge density can be specified at a charged surface. This includes essentially all the possible boundary conditions at the surface in the literature and in practice. Also, the conventional analyses are extended to the case of multiple charged surfaces. The goal is to develop a systematic method for the resolution of a PBE. This is of fundamental significance in the simulation of a dispersion of charged entities.

\section{THEORY}

The analysis is begun by considering a charged surface $\Omega$ immersed in an electrolyte solution. Under the DebyeHuckle condition, the distribution of the electrical potential is described by $(19,20)$

$$
\nabla^{2} \Psi=\Psi
$$

where $\Psi=e \Phi / k T ; \nabla^{2}$ is the Laplace operator; $\Phi$ and $\Psi$ are the electrical potential and the corresponding dimensionless form, respectively; $k$ and $e$ are, respectively, the Boltzmann constant and the elementary charge; and $T$ is the absolute temperature. We consider a general boundary condition

$$
\begin{gathered}
f(\mathbf{x}) \Psi(r, \mathbf{x})+g(\mathbf{x}) \frac{\partial \Psi(r, \mathbf{x})}{\partial \mathbf{n}}=h(\mathbf{x}), \\
r=\kappa \tilde{r} \quad(r, \mathbf{x}) \in \Omega \\
\mathbf{x}=\kappa \tilde{\mathbf{x}} \\
\kappa=\sqrt{\frac{e^{2} \sum_{i} Z_{i}^{2} n_{i 0}}{\epsilon_{\mathrm{r}} \epsilon_{0} k T}},
\end{gathered}
$$

where $Z_{i}$ and $n_{i 0}$ are, respectively, the valence and the concentration of ion species $i$ in the bulk liquid phase; $\epsilon_{\mathrm{r}}$ and $\epsilon_{0}$ are the relative permittivity of solution and the permittivity of a vacuum, respectively; and $\kappa$ denotes the reciprocal Debye length. Here, a symbol in boldface denotes a vector; $(\tilde{r}$, $\tilde{\mathbf{x}})$ and $(r, \mathbf{x})$ represent the position variable of an orthogonal coordinate and the corresponding dimensionless form, respectively, $r$ being the radial distance; $\Omega$ is the surface domain; and $\mathbf{n}$ denotes its outer normal. The position vector is decomposed into the radial distance $r$ and other coordinates $\mathbf{x}$. Under typical conditions, the solution to [1] takes the form

$$
\Psi=\sum_{n=1}^{\infty} a_{n} R_{n}(r) \Psi_{n}(\mathbf{x}),
$$

where $R_{n}$ and $\Psi_{n}$ are harmonic functions, and $a_{n}, n=1,2$, $\ldots$, are constant, which satisfy the orthogonal relation over $\Omega$

$$
\int_{\Omega} \alpha(\mathbf{x}) \Psi_{n} \Psi_{i} d \Omega= \begin{cases}0, & n \neq i \\ \text { constant, } & n=i\end{cases}
$$

where $\alpha(\mathbf{x})$ is the weighting function. If the rate of convergence of the right-hand side of [3] is sufficiently fast, it can be approximated by

$$
\Psi \approx \sum_{n=1}^{N} a_{n} R_{n}(r) \Psi_{n}(\mathbf{x}),
$$

where the coefficient $a_{n}$ needs to be determined by the associated boundary conditions. Substituting this expression into [2] yields

$$
\begin{aligned}
& f(\mathbf{x}) \sum_{n=1}^{N} a_{n} R_{n}(r) \Psi_{n}(\mathbf{x}) \\
& +g(\mathbf{x}) \sum_{n=1}^{N} a_{n} \frac{\partial R_{n}(r)}{\partial r} \Psi_{n}(\mathbf{x})=h(\mathbf{x}), \quad(r, \mathbf{x}) \in \Omega .
\end{aligned}
$$

Here, we assume that $\mathbf{n}$ is in the $r$ direction. $N$ linearly independent equations can be generated from [4] and [6] which can be used to determine $a_{n}, n=1,2, \ldots, N$. We have

$$
\mathbf{F a}-\mathbf{G a}=\mathbf{h}
$$

where

$$
\begin{aligned}
\mathbf{a} & =\left[a_{1}, a_{2}, \ldots, a_{N}\right]^{\mathrm{t}} \\
F_{i n} & =\int_{\Omega} \alpha(\mathbf{x}) R_{n}(r) f(\mathbf{x}) \Psi_{n}(\mathbf{x}) \Psi_{i}(\mathbf{x}) d \Omega \\
G_{i n} & =-\int_{\Omega} \alpha(\mathbf{x}) \frac{\partial R_{n}(r)}{\partial r} g(\mathbf{x}) \Psi_{n}(\mathbf{x}) \Psi_{i}(\mathbf{x}) d \Omega \\
h_{i} & =\int_{\Omega} \alpha(\mathbf{x}) h(\mathbf{x}) \Psi_{i}(\mathbf{x}) d \Omega
\end{aligned}
$$

In these expressions, a symbol in boldface sans serif is a matrix, the superscript $\mathrm{t}$ denotes the matrix transpose, $h_{i}$ is the $i$ th element of $\mathbf{h}$, and $F_{\text {in }}$ and $G_{i n}$ are the elements in the $i$ th row and $n$th column of $\mathbf{F}$ and $\mathbf{G}$, respectively. Solving [7] yields

$$
\mathbf{a}=(\mathbf{F}-\mathbf{G})^{-1} \mathbf{h}
$$


In practice if $N$ is large, inverting ( $\mathbf{F}-\mathbf{G}$ ) can be tedious, and may encounter some numerical difficulty. Here, a general iterative method is proposed.

\section{A. Iterative Method}

If $\mathbf{F}$ is invertible, [7] can be rewritten as

$$
\mathbf{a}=\mathbf{h}^{\prime}+\mathbf{G}^{\prime} \mathbf{a}
$$

where $\mathbf{h}^{\prime}=\mathbf{F}^{-1} \mathbf{h}$ and $\mathbf{G}^{\prime}=\mathbf{F}^{-1} \mathbf{G}$. On the other hand, if $\mathbf{F}$ is not invertible, [7] is first written as

$$
(\mathbf{F}+\mathbf{M}) \mathbf{a}=\mathbf{h}+(\mathbf{G}+\mathbf{M}) \mathbf{a},
$$

where $\mathbf{M}$ is defined such that $(\mathbf{F}+\mathbf{M})$ is invertible. The present iterative method can be summarized as below.

Step 1. Choose an arbitrary zeroth-order solutions, $\mathbf{a}_{0}$. The first-order solution, $\mathbf{a}_{1}$, is obtained by substituting $\mathbf{a}_{0}$ into the right-hand side of [9]. We have

$$
\mathbf{a}_{1}=\mathbf{h}^{\prime}+\mathbf{G}^{\prime} \mathbf{a}_{0}
$$

Step 2. Substituting $\mathbf{a}_{1}$ into the right-hand side of [9] yields the second-order solution, $\mathbf{a}_{2}$. This leads to

$$
\mathbf{a}_{2}=\mathbf{h}^{\prime}+\mathbf{G}^{\prime} \mathbf{a}_{1}=\mathbf{h}^{\prime}+\mathbf{G}^{\prime} \mathbf{h}^{\prime}+\mathbf{G}^{\prime 2} \mathbf{a}_{0} .
$$

If the above procedure is repeated, we obtain, at the $k$ th stage,

$$
\begin{aligned}
\mathbf{a}_{k}=\mathbf{h}^{\prime}+\mathbf{G}^{\prime} \mathbf{h}^{\prime}+\mathbf{G}^{\prime 2} \mathbf{h}^{\prime}+ & \cdots \\
& +\mathbf{G}^{\prime k-1} \mathbf{h}^{\prime}+\mathbf{G}^{\prime k} \mathbf{a}_{0},
\end{aligned}
$$

and, therefore,

$$
\mathbf{a}_{\infty}=\mathbf{h}^{\prime}+\mathbf{G}^{\prime} \mathbf{h}^{\prime}+\mathbf{G}^{\prime 2} \mathbf{h}^{\prime}+\mathbf{G}^{\prime 3} \mathbf{h}^{\prime}+\cdots .
$$

The sufficient and necessary condition for the right-hand side of this expression to converge is that the maximum absolute eigenvalue of $\mathbf{G}^{\prime},|\lambda(\mathbf{G})|_{\max }$, is smaller than unity (21). If this is satisfied, the solution to [7] is

$$
\begin{aligned}
\mathbf{a} & =\left(\mathbf{I}-\mathbf{G}^{\prime}\right)^{-1} \mathbf{h}^{\prime}=\left(\mathbf{I}-\mathbf{F}^{-1} \mathbf{G}\right)^{-1} \mathbf{F}^{-1} \mathbf{h} \\
& =(\mathbf{F}-\mathbf{G})^{-1} \mathbf{h},
\end{aligned}
$$

where $\mathbf{I}$ is the identity matrix. In other words, the asymptotic result of the present iterative method is the exact solution [8].

A question arises as whether $\mathbf{G}$ ' leads to a convergent solution, and if such $\mathbf{G}^{\prime}$ does not exist, can the solution procedure be modified such that a convergent solution is retrievable. Suppose that $\mathbf{G}^{\prime}$ does not lead a convergent

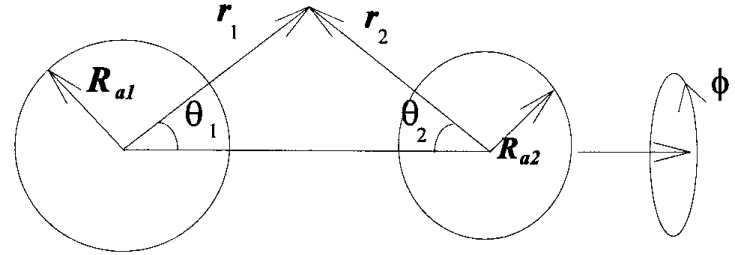

$L$

FIG. 1. Coordinates adopted for the case of two spheres.

solution. Multiplying both sides of [7] by a nonsingular matrix $\mathbf{S}$ gives

$$
\mathbf{S F a}-\mathbf{S G a}=\mathbf{S h} .
$$

This expression can be rewritten as

$$
\mathbf{a}=\mathbf{S h}+(\mathbf{I}-\mathbf{S}(\mathbf{F}-\mathbf{G})) \mathbf{a}
$$

$\mathbf{S}$ is chosen to make the maximum absolute eigenvalue of $(\mathbf{I}-\mathbf{S}(\mathbf{F}-\mathbf{G}))$ smaller than unity. If $(\mathbf{F}-\mathbf{G})$ is nonsingular, it can be shown that such an $\mathbf{S}$ exists, and can be chosen as $(\mathbf{F}-\mathbf{G})^{-1}+\epsilon \mathbf{I}$, provided that $|\epsilon|$ is sufficiently small.

\section{B. Two Identical Spheres}

Let us consider the case illustrated in Fig. 1 with $R_{a 1}=$ $R_{a 2}=R_{a}, R_{a 1}$ and $R_{a 2}$ being the radii of spheres 1 and 2, respectively. The special cases that both surfaces remain at constant potential and at constant charge density were discussed by Glendinning and Russel (14), Carnie and Chan (2), Carnie et al. (3), and Ohshima (15, 16). For the present case, [2] becomes

$$
\begin{aligned}
& f\left(\theta_{1}\right) \Psi+g\left(\theta_{1}\right) \frac{\partial \Psi}{\partial r_{1}}=h\left(\theta_{1}\right), \quad\left(r_{1}, \theta_{1}\right) \in \Omega_{1} \\
& f\left(\theta_{2}\right) \Psi+g\left(\theta_{2}\right) \frac{\partial \Psi}{\partial r_{2}}=h\left(\theta_{2}\right), \quad\left(r_{2}, \theta_{2}\right) \in \Omega_{2},
\end{aligned}
$$

where $\Omega_{1}$ and $\Omega_{2}$ represent the surfaces of spheres 1 and 2 , respectively. Here we assume that the boundary condition at the surface is independent of the $\phi$ coordinate. The general solution to [1] subject to [18a] and [18b] takes the form

$$
\begin{aligned}
\Psi=\sum_{n=1}^{\infty} a_{1 n} r_{1}^{-1 / 2} & K_{n-1 / 2}\left(r_{1}\right) P_{n-1}\left(\cos \theta_{1}\right) \\
& +\sum_{n=1}^{\infty} a_{2 n} r_{2}^{-1 / 2} K_{n-1 / 2}\left(r_{2}\right) P_{n-1}\left(\cos \theta_{2}\right)
\end{aligned}
$$


where $K_{n-1 / 2}$ and $P_{n-1}$ are the scaled modified Bessel function and the normalized Legendre function defined as, respectively,

$$
K_{n-1 / 2}(r)=\hat{K}_{n-1 / 2}(r) /\left(\hat{R}_{a}^{-1 / 2} \hat{K}_{n-1 / 2}\left(R_{a}\right)\right)
$$

and

$$
P_{n-1}(\mu)=\hat{P}_{n-1}(\mu) /\left(\int_{-1}^{1}\left(\hat{P}_{n-1}(\mu)\right)^{2} d \mu\right)^{1 / 2}
$$

In these expressions, $\hat{K}_{n-1 / 2}$ is the modified Bessel function of the second kind of order $(n-1 / 2)$, and $\hat{P}_{n-1}$ is the Legendre function of order $(n-1)(22)$. We have

$$
R_{n}(r)=r^{-1 / 2} K_{n-1 / 2}(r)
$$

and

$$
\Psi_{n}(\mathbf{x})=P_{n-1}(\cos \theta) .
$$

The symmetric nature of the problem implies that $a_{1 n}=a_{2 n}$ $=a_{n}$, and [19] and [18a] lead to

$$
\sum_{n=1}^{\infty} a_{n} \chi_{n}\left(r_{1}, \theta_{1}\right)+\sum_{n=1}^{\infty} a_{n} v_{n}\left(r_{2}, \theta_{2} \mid r_{1}, \theta_{1}\right)=h\left(\theta_{1}\right),
$$

$$
\left(r_{1}, \theta_{1}\right) \in \Omega_{1}
$$

with

$$
\begin{gathered}
\chi_{n}\left(r_{1}, \theta_{1}\right)=\left\{f\left(\theta_{1}\right) r_{1}^{-1 / 2} K_{n-1 / 2}\left(r_{1}\right)+g\left(\theta_{1}\right)\left[\frac{\partial r_{1}^{-1 / 2} K_{n-1 / 2}\left(r_{1}\right)}{\partial r_{1}}\right]\right\} P_{n-1}\left(\cos \theta_{1}\right) \\
v_{n}\left(r_{2}, \theta_{2} \mid r_{1}, \theta_{1}\right)=\left\{f\left(\theta_{1}\right) r_{2}^{-1 / 2} K_{n-1 / 2}\left(r_{2}\right) P_{n-1}\left(\cos \theta_{2}\right)+g\left(\theta_{1}\right)\left[\frac{\partial r_{2}^{-1 / 2} K_{n-1 / 2}\left(r_{2}\right) P_{n-1}\left(\cos \theta_{2}\right)}{\partial r_{1}}\right]\right\} .
\end{gathered}
$$

By referring to Fig. 1, we have

$$
\begin{aligned}
& r_{2}=\sqrt{r_{1}^{2}+L^{2}-2 r_{1} L \cos \left(\theta_{1}\right)} \\
& \theta_{2}=\sin ^{-1}\left(\frac{r_{1}}{r_{2}} \sin \theta_{1}\right)
\end{aligned}
$$

Equations [7b]-[7d] become, respectively,

$$
\begin{aligned}
h_{i} & =\int_{-1}^{1} P_{i-1}\left(\cos \theta_{1}\right) h(\theta) d \cos \theta \\
F_{i j} & =\int_{-1}^{1} P_{i-1}\left(\cos \theta_{1}\right) \chi_{j}\left(r_{1}, \theta_{1}\right) d \cos \theta
\end{aligned}
$$

and

$$
G_{i j}=-\int_{-1}^{1} P_{i-1}\left(\cos \theta_{1}\right) v_{j}\left(r_{2}, \theta_{2} \mid r_{1}, \theta_{1}\right) d \cos \theta . \quad[22 \mathrm{c}]
$$

The special cases of constant surface potential and constant surface charge density can be recovered from the present model.

\section{Two Different Spheres}

Consider the case shown in Fig. 1 with $R_{a 1} \neq R_{a 2}$. We assume that the boundary conditions at the surface associated with [1] are

$$
\begin{aligned}
& f_{1}\left(\theta_{1}\right) \Psi+g_{1}\left(\theta_{1}\right) \frac{\partial \Psi}{\partial r_{1}}=h_{1}\left(\theta_{1}\right), \quad\left(r_{1}, \theta_{1}\right) \in \Omega_{1} \\
& f_{2}\left(\theta_{2}\right) \Psi+g_{2}\left(\theta_{2}\right) \frac{\partial \Psi}{\partial r_{2}}=h_{2}\left(\theta_{2}\right), \quad\left(r_{2}, \theta_{2}\right) \in \Omega_{2} .
\end{aligned}
$$

The general solution of [1] can be expressed as [19]. In this case, $a_{1 n} \neq a_{2 n}, n=1,2,3, \ldots$ Substituting [19] into [23] yields

$$
\begin{aligned}
& \sum_{n=1}^{\infty} a_{1 n} \chi_{n}\left(r_{1}, \theta_{1}\right)+\sum_{n=1}^{\infty} a_{2 n} v_{n}\left(r_{2}, \theta_{2} \mid r_{1}, \theta_{1}\right)=h_{1}\left(\theta_{1}\right) \\
& \sum_{n=1}^{\infty} a_{1 n} v_{n}\left(r_{1}, \theta_{1} \mid r_{2}, \theta_{2}\right)+\sum_{n=1}^{\infty} a_{2 n} \chi_{n}\left(r_{2}, \theta_{2}\right)=h_{2}\left(\theta_{2}\right),
\end{aligned}
$$

where $\chi_{n}$ and $v_{n}$ are defined in [21a] and [21b], respectively. Expressing these relations in the form of [7], we obtain

$$
\begin{aligned}
\mathbf{F}_{1} \mathbf{a}_{1}-\mathbf{G}_{1} \mathbf{a}_{2} & =\mathbf{h}_{1} \\
-\mathbf{G}_{2} \mathbf{a}_{1}+\mathbf{F}_{2} \mathbf{a}_{2} & =\mathbf{h}_{2} .
\end{aligned}
$$

These expressions lead to

$$
\begin{aligned}
& \mathbf{a}_{1}=\mathbf{I} \mathbf{h}_{1}^{\prime}+\mathbf{G}_{12} \mathbf{a}_{2} \\
& \mathbf{a}_{2}=\mathbf{I} \mathbf{h}_{2}^{\prime}+\mathbf{G}_{21} \mathbf{a}_{1},
\end{aligned}
$$


where $\mathbf{h}_{1}^{\prime}=\mathbf{F}_{1}^{-1} \mathbf{h}_{1}, \mathbf{h}_{2}^{\prime}=\mathbf{F}_{2}^{-1} \mathbf{h}_{2}, \mathbf{G}_{12}=\mathbf{F}_{1}^{-1} \mathbf{G}_{1}$, and $\mathbf{G}_{21}=$ $\mathbf{F}_{2}^{-1} \mathbf{G}_{2}$. Substituting [29] into [28] gives

$$
\mathbf{a}_{1}=\mathbf{l h}{ }_{1}^{\prime}+\mathbf{G}_{12} \mathbf{h}_{2}^{\prime}+\mathbf{G}_{12} \mathbf{G}_{21} \mathbf{a}_{1}
$$

Applying the present iterative procedure, we obtain

$$
\begin{aligned}
\mathbf{a}_{1}=\mathbf{l} \mathbf{h}_{1}^{\prime}+\mathbf{G}_{12} \mathbf{h}_{2}^{\prime}+\mathbf{G}_{12} \mathbf{G}_{21} \mathbf{h}_{1}^{\prime} & \\
& +\mathbf{G}_{12} \mathbf{G}_{21} \mathbf{G}_{12} \mathbf{h}_{2}^{\prime}+\cdots
\end{aligned}
$$

Similarly,

$$
\begin{aligned}
\mathbf{a}_{2}=l \mathbf{h}_{2}^{\prime}+\mathbf{G}_{21} \mathbf{h}_{1}^{\prime}+\mathbf{G}_{21} \mathbf{G}_{12} \mathbf{h}_{2}^{\prime} & \\
& +\mathbf{G}_{21} \mathbf{G}_{12} \mathbf{G}_{21} \mathbf{h}_{1}^{\prime}+\cdots .
\end{aligned}
$$

The first term on the right-hand side of [31] can be interpreted as the influence of surface 1 on itself. The second term represents the direct influence of surface 2 on surface 1 , the third term is an indirect influence of surface 1 on itself, etc. Thus, $\mathbf{G}_{i j}$ can be viewed as a signal transfer matrix from surface $j$ to surface $i$. If the influence of a surface to the other is small, then $\left|\lambda\left(\mathbf{G}_{i j}\right)\right|_{\max } \ll 1$. The solution to [26] and [27] can be approximated by

$$
\begin{aligned}
& \mathbf{a}_{1}=\mathbf{h}_{1}^{\prime}+\mathbf{G}_{12} \mathbf{h}_{2}^{\prime}+\mathbf{G}_{12} \mathbf{G}_{21} \mathbf{h}_{1}^{\prime}, \\
& \mathbf{a}_{2}=\mathbf{h}_{2}^{\prime}+\mathbf{G}_{21} \mathbf{h}_{1}^{\prime}+\mathbf{G}_{21} \mathbf{G}_{12} \mathbf{h}_{2}^{\prime} .
\end{aligned}
$$

Here, $\mathbf{G}_{i j}$ is a function of the radii of particles, the relative position between them, and the boundary conditions specified at the surfaces. The right-hand sides of [31] and [32] converge if $\left|\lambda\left(\mathbf{G}_{12} \mathbf{G}_{21}\right)\right|_{\max }<1$. It can be shown that if $\left\|\mathbf{G}_{12}\right\|_{s}<1$ and $\left\|\mathbf{G}_{21}\right\|_{s}<1$, this is satisfied (Appendix A), where $\|\mathbf{M}\|_{s}$ denotes the maximal singular value of $\mathbf{M}$.

\section{Multiple Identical Spheres}

The result for two spheres can be extended directly to multiple spheres. For $S$ identical spheres with relative position between any two shown in Fig. 8, we have, on the basis of [24]-[32],

$$
\mathbf{a}_{i}=\sum_{n=1}^{\infty} \mathbf{E}_{n}
$$

where $\mathbf{E}_{n}$ is the $n$th order contribution to $\mathbf{a}_{i}$ defined by

$$
\begin{array}{ll}
\mathbf{E}_{0}= & \mathbf{h}_{i}^{\prime} \\
\mathbf{E}_{1}= & \sum_{\substack{j=1 \\
j \neq i}}^{S} \mathbf{G}_{i j} \mathbf{h}_{j}^{\prime} \\
\mathbf{E}_{2}= & \sum_{\substack{x=1 \\
x \neq i}}^{S} \sum_{\substack{j=1 \\
j \neq x}}^{S} \mathbf{G}_{i x} \mathbf{G}_{x j} \mathbf{h}_{j}^{\prime} \\
\mathbf{E}_{3}= & \sum_{\substack{x=1 \\
x \neq i}}^{S} \sum_{\substack{y=1 \\
y \neq x}}^{S} \mathbf{G}_{i x} \mathbf{G}_{x y} \mathbf{G}_{y j} \mathbf{h}_{j}^{\prime}
\end{array}
$$

$p$ times

$$
\mathbf{E}_{p}=\overbrace{\sum_{\substack{x=1 \\ x \neq i}}^{S} \sum_{\substack{y=1 \\ y \neq x}}^{S} \sum_{\substack{z=1 \\ z \neq y}}^{S} \cdots \sum_{\substack{j=1 \\ j \neq w}}^{S} \mathbf{G}_{i x} \mathbf{G}_{x y} \mathbf{G}_{y z} \cdots \mathbf{G}_{w j} \mathbf{h}_{j}^{\prime}}^{\underbrace{}_{j=w}}
$$

A sufficient condition for [34] to converge is (Appendix A)

$$
\left\|\mathbf{G}_{x y}\right\|_{s}<\frac{1}{S-1}, \quad x, y=1,2, \ldots, S, x \neq y .
$$

It should be pointed out that this is a conservative condition, and is satisfied for the case of a dilute system. In this case, [34] can be approximated by

$$
\mathbf{a}_{i}=\mathbf{h}_{i}^{\prime}+\sum \mathbf{G}_{i j} \mathbf{h}_{j}^{\prime}+\sum \sum \mathbf{G}_{i x} \mathbf{G}_{x j} \mathbf{h}_{j}^{\prime}+\cdots
$$

where $\mathbf{G}_{i j}$ and $\mathbf{h}_{j}^{\prime}$ are defined in Appendix B. Here, $\mathbf{G}_{i j}$ is a function of the orientations of spheres $i$ and $j$, and $\mathbf{h}^{\prime}$ is a function of boundary condition. In $f, g$, and $h$ in [2] are constant, $\mathbf{G}_{i j}$ is a function of the center-to-center vector defined by spheres $i$ and $j$ only. The performance of the present iterative method depends largely on whether $\mathbf{G}_{i j}$ and $\mathbf{h}_{j}^{\prime}$ can be calculated efficiently. In any event, direct inversion of a large matrix (if $S$ is large), if [8] is adopted, can be avoided.

\section{RESULT AND DISCUSSION}

The applicability of the present iterative method is examined by three examples. In the first example we consider the spherical surface shown in Fig. 2. The following boundary condition is assumed:

$$
f(\cos \theta) \Psi+g(\cos \theta) \frac{\partial \Psi}{\partial r}=h(\cos \theta), \quad r=R_{a} .
$$

A charge-regulated surface may lead to this type of boundary condition. For instance, the surface potential $\Psi_{\mathrm{S}}$ and the 


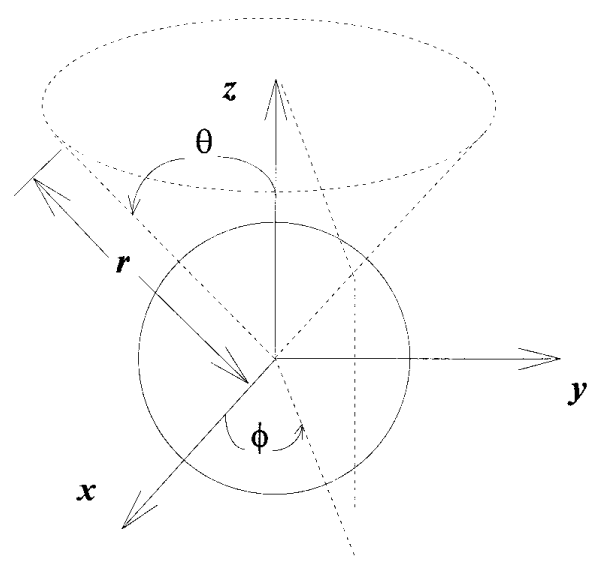

FIG. 2. Coordinates adopted for the case of single sphere.

surface charge density $\rho_{\mathrm{S}}$ for a spherical charge-regulated surface are related by (7)

$$
\frac{\rho_{\mathrm{S}}(\cos \theta)}{-\epsilon_{\mathrm{r}} \epsilon_{0} \kappa k T}=\frac{\partial \Psi}{\partial r}=N(\Psi)
$$

where

$$
N(\Psi)=C \frac{\delta \sinh \left(\Psi_{N}-\Psi_{\mathrm{S}}\right)}{1+\delta \cosh \left(\Psi_{N}-\Psi_{\mathrm{S}}\right)}
$$

$C, \delta$, and $\Psi_{N}$ are constant. If [39a] is linearized around $\Psi_{0}(\cos \theta)$, we have

$$
\left.\frac{\partial N(\Psi)}{\partial \Psi}\right|_{\Psi=\Psi_{0}} \Psi+\frac{\partial \Psi}{\partial r}=-N\left(\Psi_{0}\right)+\left.\frac{\partial N(\Psi)}{\partial \Psi}\right|_{\Psi=\Psi_{0}} \Psi_{0},
$$

which is of the form of [38]. The solution to [1] subject to [40] is [19] without the second term on its right-hand side. Figure 3 shows the assumed variations of $f, g$, and $h$ in [38], and the distribution of the dimensionless electrical potential is illustrated in Fig. 4. The value of $N$ in [3] is 10, and the variation of $a_{n}$ is presented in Fig. 5. In this example, $\left|\lambda\left(\mathbf{F}^{-1} \mathbf{G}\right)\right|_{\max }=35.737>1$ and $\left|\lambda\left(\mathbf{G}^{-1} \mathbf{F}\right)\right|_{\max }=0.4924$ $<1$. This implies that care must be taken in searching for a convergent solution for a general mixed boundary-valued problem. In this example, [9] must be rewritten as

$$
\mathbf{a}=-\mathbf{G}^{-1} \mathbf{h}+\mathbf{G}^{-1} \mathbf{F a} .
$$

If both $\left|\lambda\left(\mathbf{F}^{-1} \mathbf{G}\right)\right|_{\max }$ and $\left|\lambda\left(\mathbf{G}^{-1} \mathbf{F}\right)\right|_{\max }$ are greater than unity, [16] and [17] need to be employed. As can be seen from Fig. 5, the rate of convergence of the right-hand side of [3] is fast, and [5] is appropriate. The charge-regulated model has been examined extensively in the literature (e.g., $(1-7))$. The approaches can be classified roughly into two

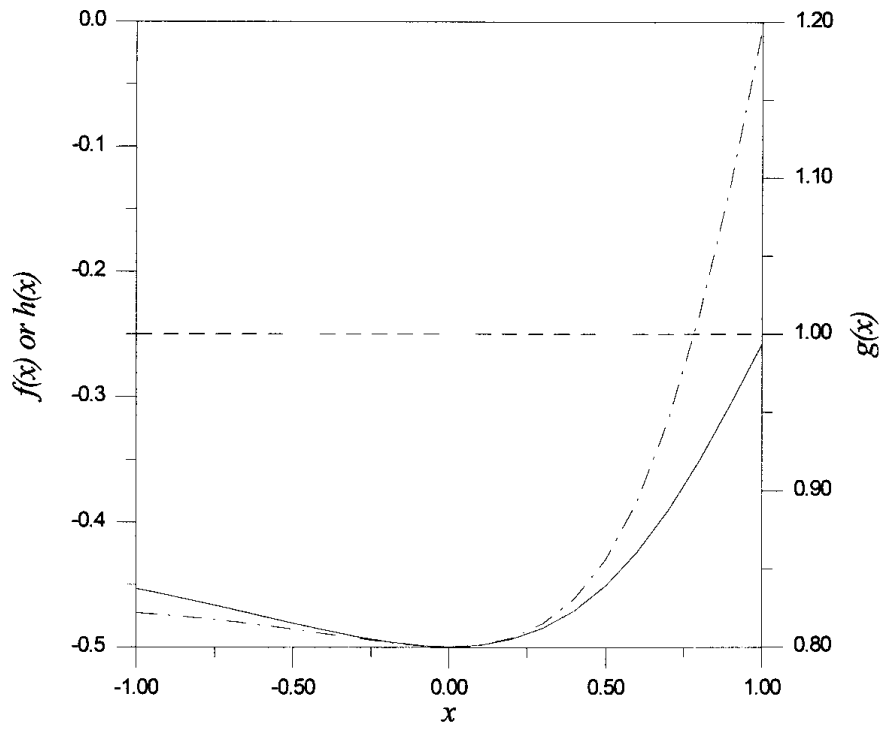

FIG. 3. Assumed variations for $f(x),-; g(x),---$, and $h(x),-\cdots$, in the numerical simulation.

categories: analytical (e.g., orthogonal expansion) and numerical (e.g., finite element) methods. The former is adopted mainly for linear governing equations and linear boundary conditions, and the latter for nonlinear governing equations and nonlinear boundary conditions. An orthogonal expansion method which assumed that both $f(r, \mathbf{x})$ and $g(r, \mathbf{x})$ in [2] are constant $(1,3,7)$ was used. The key step of this approach is the transformation expressed in [41], which simplifies significantly the analysis. If $f(r, \mathbf{x})$ and/or $g(r$, $\mathbf{x})$ are not constant, e.g., they are functions of angular position, the orthogonal expansion technique is inapplicable since [41] will lead to a more complicated problem than the original one. On the other hand, this will not cause any problem in the present analysis.

The second example is shown in Fig. 1. Here, we consider two different spherical surfaces. The contours of the electrical potential distributions for two different center-to-center

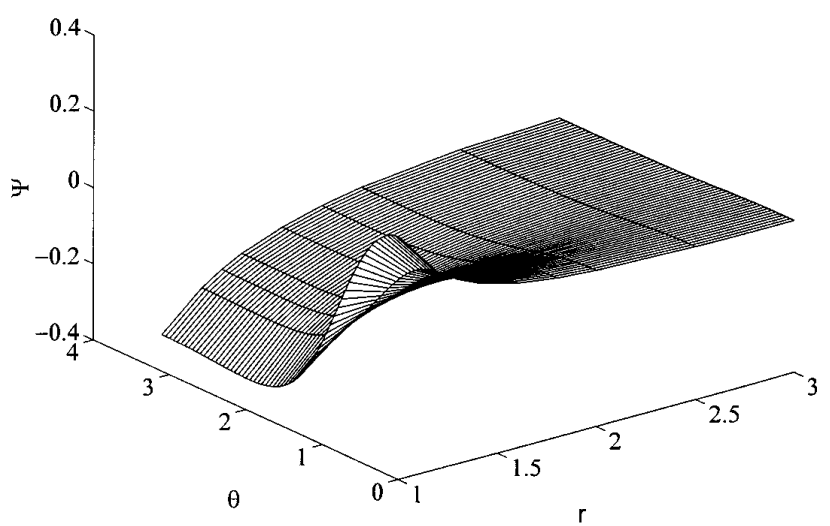

FIG . 4. Distribution of the dimensionless electrical potential at surface for the case of Fig. 3 with $N=10$ in [3]. 


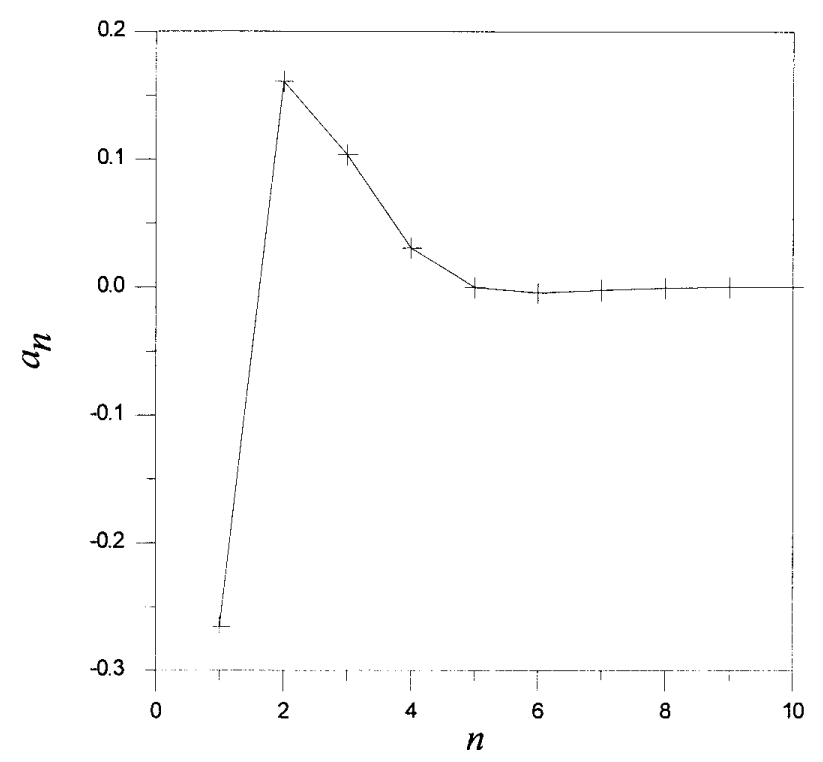

FIG. 5. Variation of the coefficient $a_{n}$ in [3] for the case of Fig. 4.

distances $L$ are illustrated in Figs. 6 and 7. These figures suggest that, for the same number of iterations, the greater the $L$, the better the performance of the present method. This is because $\left|\lambda\left(\mathbf{G}_{12} \mathbf{G}_{21}\right)\right|_{\text {max }}$ decreases with the increase in $L$. In other words, the influence of a surface on the other diminishes with the increase in $L$, as expected. In this example, the evaluations of $\mathbf{G}_{1}$ and $\mathbf{G}_{2}$ through the surface integrals defined in [28] and [29] are tedious. This difficulty can be circumvented by replacing $r_{2}^{-1 / 2} K_{n-1 / 2}\left(r_{2}\right) P_{n-1}\left(\cos \theta_{2}\right)$ with the infinite series $\sum_{m=1}^{\infty} r_{1}^{-1 / 2} I_{m-1 / 2}\left(r_{1}\right) P_{m-1}\left(\cos \theta_{1}\right)$, and $r_{1}^{-1 / 2} K_{n-1 / 2}\left(r_{1}\right) P_{n-1}\left(\cos \theta_{1}\right)$ by $\sum_{m=1}^{\infty} r_{2}^{-1 / 2} I_{m-1 / 2}\left(r_{2}\right) P_{m-1}$ $\left(\cos \theta_{2}\right)$, where $I_{m-1 / 2}$ is the modified Bessel function of the

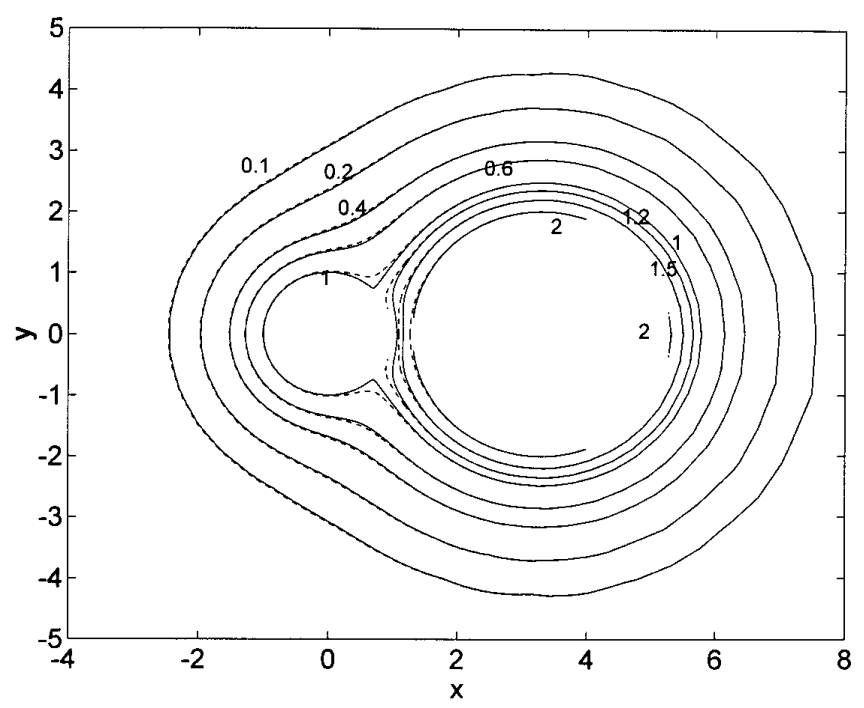

FIG . 6. Contours of the electrical potential distribution for the case of two spherical surfaces. Key: $R_{a 1}=1 ; R_{a 2}=2 ; L=3.3 ; \Psi_{\mathrm{S}, 1}=1$; and $\Psi_{\mathrm{S}, 2}$ $=2 ;-$, results based on [8];---, results based on [31] and [32].

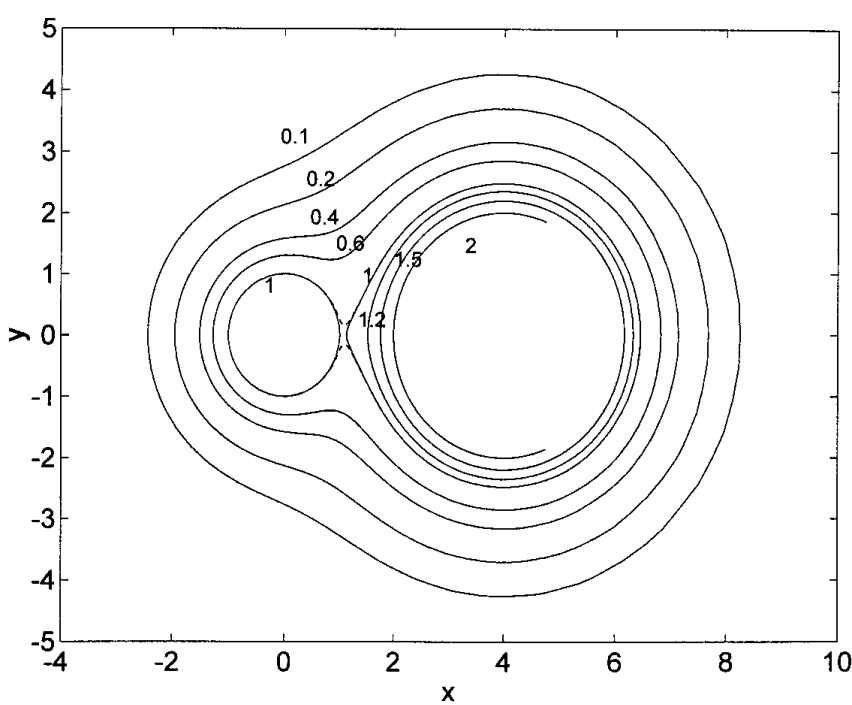

FIG. 7. Contours of the electrical potential distribution for the case of two spherical surfaces. Key: Same as Fig. 6, except that $L=4$.

first kind of order $(m-1 / 2)(14)$. This takes the advantage that $P_{m-1}\left(\cos \theta_{1}\right)$ and $P_{m-1}\left(\cos \theta_{2}\right), m=1,2, \ldots$, are orthogonal functions on $\Omega_{1}$ and on $\Omega_{2}$, respectively. For the case shown in Fig. 1, the harmonic function $r_{2}^{-1 / 2}$ $\hat{K}_{n+1 / 2}\left(r_{2}\right) \hat{P}_{n}\left(\cos \theta_{2}\right)$ can be expressed in terms of $\left(r_{1}, \theta_{1}\right)$ as (14)

$$
\begin{array}{r}
r_{2}^{-1 / 2} \hat{K}_{n+1 / 2}\left(r_{2}\right) \hat{P}_{n}\left(\cos \theta_{2}\right) \\
=\sum_{m=0}^{\infty}(2 m+1) B_{n m} r_{1}^{-1 / 2} \hat{I}_{m+1 / 2}\left(r_{1}\right) \hat{P}_{m}\left(\cos \theta_{1}\right) \\
n=0,1, \ldots \\
n=
\end{array}
$$

where

$$
\begin{gathered}
B_{n m}=\sum_{v=0}^{\infty} A_{n m}^{v}(\pi / 2) L^{-1 / 2} \hat{K}_{n+1 / 2}(L) \\
\Gamma(n-v+1 / 2) \Gamma(m-v+1 / 2) \\
\times \Gamma(v+1 / 2)(n+m-v) ! \\
\times(n+m-2 v+1 / 2)
\end{gathered} .
$$

$\Gamma(x)$ is the Gamma function.

In the third example, a system that comprises three identical spheres is considered. Two cases are examined: the distance between any two spheres is the same, and the relative positions of spheres are arbitrary. The performance of the present iterative method for these cases is illustrated in Figs. 9 and 10. In the first case, $\left\|\mathbf{G}_{12}\right\|_{s}=\left\|\mathbf{G}_{13}\right\|_{s}=\left\|\mathbf{G}_{23}\right\|_{s}=0.2854$ $<0.5$, and [36] is satisfied. Figure 9 shows that the rate of convergence of the iterative solution is very fast: the devia- 


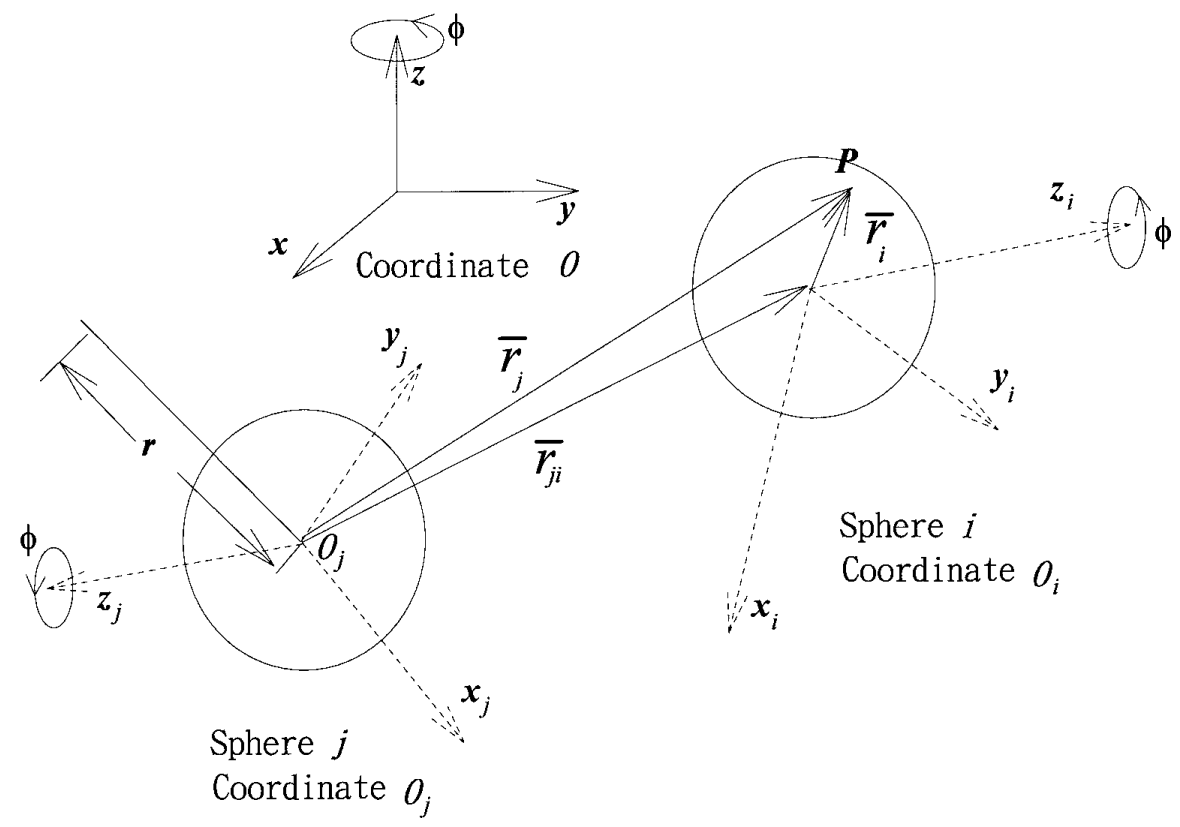

FIG. 8. Coordinates adopted for the case of multiple surfaces.

tion of the third-order solution is on the order of $5 \%$. In the case of Fig. 10, $\left\|\mathbf{G}_{12}\right\|_{s}=0.2842,\left\|\mathbf{G}_{13}\right\|_{s}=0.0288$, and $\left\|\mathbf{G}_{23}\right\|_{s}$ $=0.2042$, and [36] is satisfied. A conclusion similar to that based on Fig. 9 can be drawn from Fig. 10. Note that, since the average center-to-center distance between two spheres is greater than that in Fig. 9, the rate of convergence is a

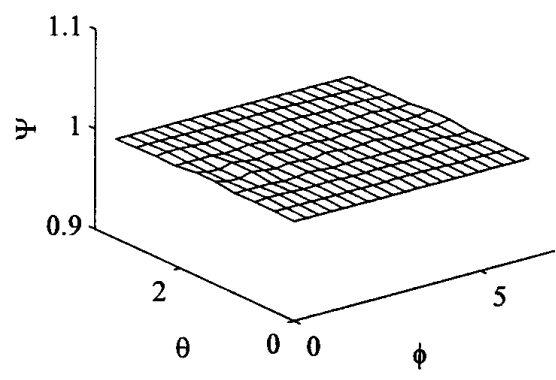

C

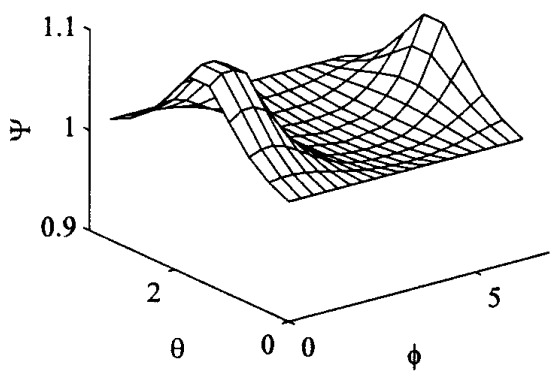

b

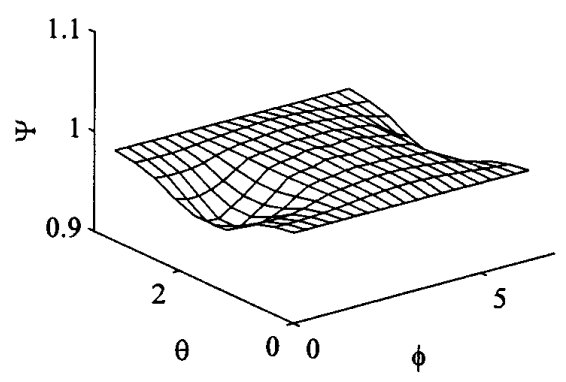

d

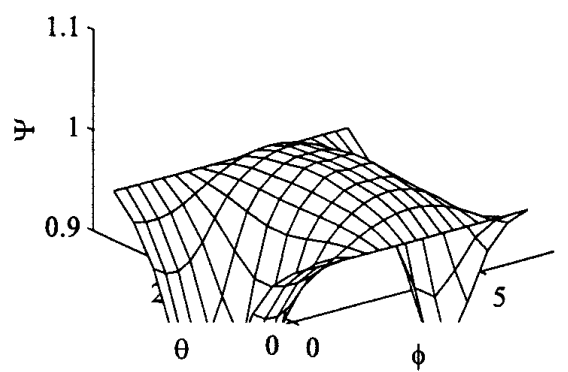

FIG. 9. Performance of the present iterative method for the case of three identical spheres with $R_{a}=1, \Psi_{\mathrm{S}}=1$, and $Q=5$ in $[\mathrm{B} 2$ ]. The centers are located at $(0,0,0),(1.25,1.25 \sqrt{3}, 0)$, and $(2.5,0,0)$, the center-to-center distance between any two spheres is the same. Only the result for one of the spheres is shown. (a) Result based on [8]; (b) third-order solution; (c) second-order solution; (d) first-order solution. 
a
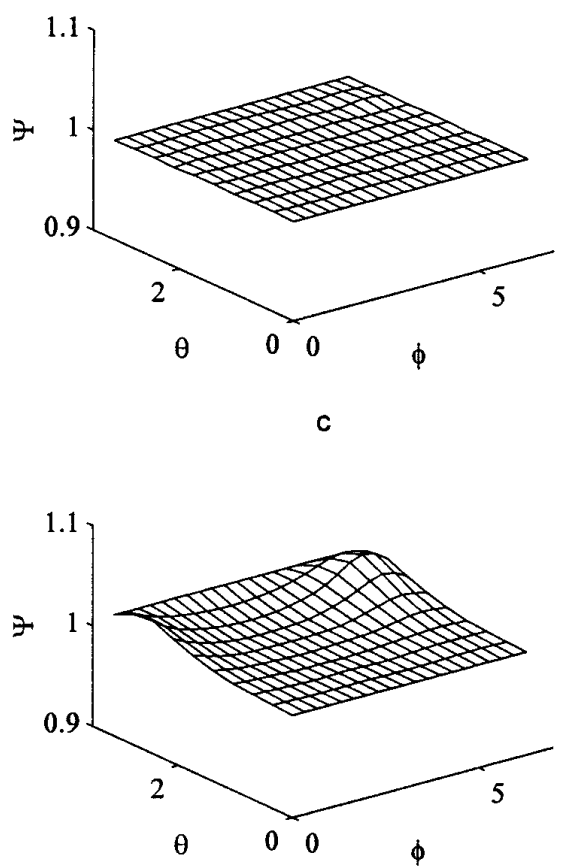

b
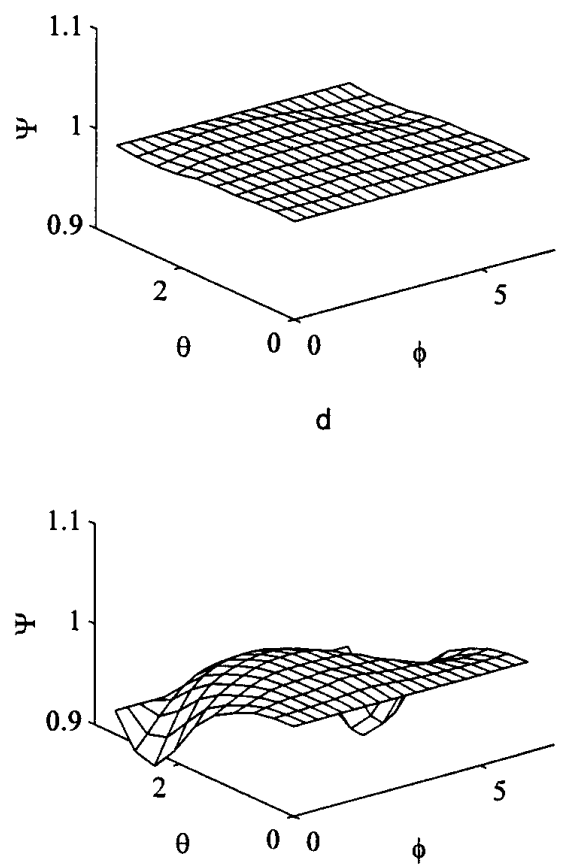

FIG. 10. Performance of the present iterative method for the case of three identical spheres with $R_{a}=1, \Psi_{\mathrm{S}}=1$, and $Q=5$ in [B2]. The centers are located arbitrarily at $(0,3,2),(1.25,1.25 \sqrt{3}, 0)$, and $(2.5,0,1)$. Only the result for one of the spheres is shown. (a) Result based on [8]; (b) third-order solution; (c) second-order solution; (d) first-order solution.

faster. It should be pointed out that, although a multipole expansion technique has been used widely in the area of fluid mechanics (e.g., $(25,26))$, it was adopted only for the case of two particles (e.g., $(1,3,14-17))$ in the discussion of charged surfaces in electrolyte solutions. This is because [41] is applicable in this case. If more than two particles are considered, the electrical potential is a function of azimuthal position, and the transformation, [41], becomes meaningless. On the other hand, the present iterative method is still applicable.

As shown in [14] and [15], the present iterative method can be viewed as an expansion of $\left(\mathbf{I}-\mathbf{G}^{\prime}\right)$ into an infinite series. It is equivalent to the reflection method often adopted for the case of two interacting surfaces. For example, [41] was used by Ohshima (15) to obtain an explicit analytic solution for the case of two spherical surfaces at constant potential/charge density in the form of an infinite series. The same result can be derived from [31] and [32]. To obtain an explicit solution through the reflection method, however, requires expressing the coordinates of one surface to those of the other. This is extremely tedious, if not impossible, for the cases of irregular surfaces, multiple surfaces, and mixed boundary condition problems. In a previous study (23), [6] was solved for the case of a single charged surface on the basis of minimum two-norm. The treatment expressed in [7] and [8], on the other hand, is a weighted residual or
Ritz method with weighting function $\Psi_{i}(\mathbf{x})$, an approach often employed in finite element (24).

As shown in [15], the asymptotic result of the present iterative method is the same as that obtained by a direct matrix inversion, [8]. As the number of charged surfaces $S$ increases the latter becomes inefficient due to the difficulty of inverting a large matrix, and can be inaccurate due to round-off errors. On the other hand, these problems are not present in the former. The matrix dimension involved in the present iterative method depends on the dimension of the signal transfer matrix $\mathbf{G}_{i j}$, not on $S$, a highly desirable property. Furthermore, solving [7] by taking matrix inversion, although giving a solution directly, provides no physical meaning. In contrast, the present iterative procedure yields new insights into the problem under consideration. These include the following: (i) A signal transfer matrix $\mathbf{G}_{i j}$, the most significant parameter of the problem, is defined, and a multiple-particles problem reduces to a two-particles problem. (ii) A criterion is proposed to decide whether the separation distances between surfaces are appropriate for various approximate procedures. For example, if any two surfaces are separated far enough, since $\left\|\mathbf{G}_{i j}\right\|_{s}$ is small, [37] can be approximated by

$$
\mathbf{a}_{i} \approx \mathbf{h}_{i}+\sum \mathbf{G}_{i j} \mathbf{h}_{j} .
$$


In this case, the dimensionless surface potential of the $i$ th sphere, $\Psi_{\mathrm{S}, i}$ and the corresponding dimensionless surface charge density, $\rho_{\mathrm{S}, i}$, can be calculated by, respectively,

$$
\Psi_{\mathrm{S}, i} \approx \sum \mathbf{L}_{i j} \mathbf{a}_{j}
$$

and

$$
\rho_{\mathrm{S}, i} \approx \sum \frac{-\partial \mathbf{L}_{i j}}{\partial r_{i}} \mathbf{a}_{j}=\sum \mathbf{S}_{i j} \mathbf{a}_{j}
$$

where

$$
\begin{aligned}
L_{i j} & =\left[R_{1}\left(r_{j}\right) \Psi_{1}\left(\cos \left(\theta_{j}\right), \phi_{j}\right), R_{2}\left(r_{j}\right)\right. \\
& \left.\times \Psi_{2}\left(\cos \left(\theta_{j}\right), \phi_{j}\right), \ldots, R_{N}\left(r_{j}\right) \Psi_{N}\left(\cos \left(\theta_{j}\right), \phi_{j}\right)\right] .
\end{aligned}
$$

Here $R_{n}\left(r_{j}\right)$ and $\Psi_{n}\left(\cos \left(\theta_{j}\right), \phi_{j}\right), n=1,2, \ldots, N$, are defined in Appendix $\mathrm{B}$, and $r_{j}, \theta_{j}$, and $\phi_{j}$ are defined in [B11]. $\mathbf{L}_{i j}$ and $\mathbf{S}_{i j}$ can be interpreted, respectively, as a potential transfer matrix and a charge transfer matrix from the $j$ th surface to the $i$ th surface. Since the influence of a surface on itself is greater than that on the others, $\left\|\mathbf{L}_{i j}\right\|_{s},\left\|\mathbf{S}_{i j}\right\|_{s}$, and $\left\|\mathbf{G}_{i j}\right\|_{s}$ are small, and are of order $o^{1}$. Substituting [42] into [43] and [44], we obtain

$$
\begin{aligned}
\Psi_{i} & =\mathbf{L}_{i i} \mathbf{h}_{i}+\sum_{j \neq i}\left(\mathbf{L}_{i i} \mathbf{G}_{i j}+\mathbf{L}_{i j}\right) \mathbf{h}_{j}+o^{2} \\
\rho_{i} & =\mathbf{S}_{i i} \mathbf{h}_{i}+\sum_{j \neq i}\left(\mathbf{S}_{i i} \mathbf{G}_{i j}+\mathbf{S}_{i j}\right) \mathbf{h}_{j}+o^{2} .
\end{aligned}
$$

The first and the second terms on the right-hand sides of these expression denote, respectively, the zeroth-order and the first-order approximations. The electrical interaction free energy of the $i$ th surface for the case of constant potential is

$$
\begin{aligned}
V_{\mathrm{e}} & =\left(\frac{-1}{2} \int \Psi_{\mathrm{S}, i} \rho_{\mathrm{S}, i} d \Omega_{i}\right)-\left(\frac{-1}{2} \int \Psi_{\mathrm{S}, i} \rho_{\mathrm{S}, i}(\infty) d \Omega_{i}\right) \\
& =\sum_{j \neq i} \frac{-1}{2} \Psi_{\mathrm{S}, i} \int\left(\mathbf{S}_{i i} \mathbf{G}_{i j} \mathbf{h}_{j}+\mathbf{S}_{i j} \mathbf{h}_{j}\right) d \Omega_{i},
\end{aligned}
$$

and for the case of constant charge density is

$$
\begin{aligned}
V_{\mathrm{e}} & =\left(\frac{1}{2} \int \Psi_{\mathrm{S}, i} \rho_{\mathrm{S}, i} d \Omega_{i}\right)-\left(\frac{1}{2} \int \Psi_{\mathrm{S}, i}(\infty) \rho_{\mathrm{S}, i} d \Omega_{i}\right) \\
& =\sum_{j \neq i} \frac{1}{2} \rho_{\mathrm{S}, i} \int\left(\mathbf{L}_{i i} \mathbf{G}_{i j} \mathbf{h}_{j}+\mathbf{L}_{i j} \mathbf{h}_{j}\right) d \Omega_{i} .
\end{aligned}
$$

These expressions imply that the interaction energy of the system under consideration can be estimated in a pairwiseaddition manner. Furthermore, if $\left\|\mathbf{G}_{i j}\right\|_{s} \ll 1$, then

$$
\mathbf{a}_{i} \approx \mathbf{h}_{i}
$$

Equations [43] and [50] suggest that a linear superposition approximation is satisfactory. (iii) If the condition shown in [36] is satisfied, a system containing a large number of particles can be simulated by one which has relatively few particles. This is because if the $i$ th and the $j$ th surfaces are separated far enough, $\left\|\mathbf{G}_{i j}\right\|_{s}$ is small, and the contribution of the latter to the former in [35] can be neglected.

\section{APPENDIX A}

The singular value of $\mathbf{G}$ with dimension $m \times n, \sigma$, is defined as the positive square root of the eigenvalue of $\mathbf{G}^{\mathbf{t}} \mathbf{G}$, if $m \geqslant n$, or $\mathbf{G G}^{\mathrm{t}}$, if $m \leqslant n$. The maximum and the minimum singular values of $\mathbf{G}$ are denoted as $\|\mathbf{G}\|_{s}$ and $\|\mathbf{G}\|_{i}$, respectively. $\|\mathbf{G}\|_{s}$ satisfies (27)

$$
\|\mathbf{G}\|_{s}=\sup _{x \neq 0} \frac{\|\mathbf{G} \mathbf{x}\|}{\|\mathbf{x}\|}
$$

where $\|\mathbf{x}\|$ is the two-norm of $\mathbf{x}$; i.e., $\|\mathbf{x}\|=\sqrt{\sum_{i} x_{i}^{2}}, x_{i}$ being the $i$ th component of $\mathbf{x}$. The absolute eigenvalue of $\mathbf{G}$, $|\lambda(\mathbf{G})|$, is bounded by $\|\mathbf{G}\|_{i}$ and $\|\mathbf{G}\|_{s}$, i.e.,

$$
\|\mathbf{G}\|_{i} \leqslant|\lambda(\mathbf{G})| \leqslant\|\mathbf{G}\|_{s}
$$

Furthermore, if $\mathbf{G}$ and $\mathbf{H}$ are dimensional compatible ( $\mathbf{G H}$ exists ), then

$$
\|\mathbf{G H}\|_{s} \leqslant\|\mathbf{G}\|_{s}\|\mathbf{H}\|_{s}
$$

Let us consider the expression

$$
\mathbf{T}_{i j}=\sum_{\substack{x=1 \\ x \neq i}}^{S} \sum_{\substack{y=1 \\ y \neq x}}^{S} \sum_{\substack{z=1 \\ z \neq y}}^{S} \cdots \sum_{\substack{j=1 \\ j \neq w}}^{S} \mathbf{G}_{i x} \mathbf{G}_{x y} \mathbf{G}_{y z} \cdots \mathbf{G}_{w j}
$$

Suppose that $\left\|\mathbf{G}_{i j}\right\|_{s}<\epsilon, i, j=1,2, \ldots, S$, and $\epsilon$ is some small positive number. If there are $k$ summations on the right-hand side of [A4], then [A3], [A4], and the inequality

$$
\|\mathbf{G}+\mathbf{H}\|_{s} \leqslant\|\mathbf{G}\|_{s}+\|\mathbf{H}\|_{s}
$$

imply that

$$
\left\|\mathbf{T}_{i j}\right\|_{s} \leqslant(S-1)^{k} \epsilon^{k}
$$

Therefore, if $\epsilon<1 /(S-1)$, then $\left\|\mathbf{T}_{i j}\right\|_{s}<1$. 


\section{APPENDIX B}

Consider a charged sphere with boundary condition at the surface

$$
f(\mu, \phi) \Psi+g(\mu, \phi) \frac{\partial \Psi}{\partial r}=h(\mu, \phi)
$$

immersed in an electrolyte solution. The distribution of the dimensionless electrical potential can be expressed as

$$
\begin{aligned}
\Psi(r, \theta, \phi)= & \sum_{q=0}^{\infty} \sum_{p=-q}^{q} \alpha_{p q} r^{-1 / 2} K_{q+1 / 2}(r) P_{q}^{|p|} \\
& \times(\cos \theta) \exp (i p \phi) \\
\approx & \sum_{q=0}^{Q} \sum_{p=-q}^{q} \alpha_{p q} r^{-1 / 2} K_{q+1 / 2}(r) P_{q}^{|p|} \\
& \times(\cos \theta) \exp (i p \phi),
\end{aligned}
$$

where $i=\sqrt{-1}$. This expression can be rewritten as

$$
\Psi=\sum_{n=1}^{N} a_{n} R_{n}(r) \Psi_{n}(\mu, \phi),
$$

where $\mu=\cos (\theta), a_{n}=\alpha_{p q}, n=\sum_{k=0}^{q-1}(2 k+1)+(p+$ $q+1), R_{n}=K_{q+1 / 2}(r)$, and $\Psi_{n}=P_{q}^{|p|}(\cos \theta) \exp (i p \phi)$. We define

$$
\begin{aligned}
\mathbf{G}_{i j} & =\mathbf{F}^{-1} \mathbf{G} \\
\mathbf{h}_{j}^{\prime} & =\mathbf{F}^{-1} \mathbf{h}_{j} .
\end{aligned}
$$

The elements of $\mathbf{F}$ and $\mathbf{G}$ are defined by

$$
\begin{aligned}
F_{m n}= & \int_{-1}^{1} \int_{0}^{2 \pi} \chi_{m}\left(r_{i}, \mu_{i}, \phi_{i}\right) \Psi_{m}^{*}\left(\mu_{i}, \phi_{i}\right) d \phi_{i} d \mu_{i}, \\
G_{m n}= & \left.-\int_{-1}^{1} \int_{0}^{2 \pi} v_{n}\left(\mu_{i}, \phi_{i}\right) \in \Omega_{i}, \phi_{j} \mid r_{i}, \mu_{i}, \phi_{i}\right) \Psi_{m}^{*}\left(\mu_{i}, \phi_{i}\right) \\
& \times d \phi_{i} d \mu_{i}, \quad m, n=1,2, \ldots, N
\end{aligned}
$$

where the superscript $*$ denotes the complex conjugate, and

$$
\begin{aligned}
& \chi_{n}\left(r_{1}, \theta_{1}\right)=\left\{f\left(\mu_{i}, \phi_{i}\right) R_{n}\left(r_{i}\right)+g\left(\mu_{i}, \phi_{i}\right)\right. \\
& \left.\times\left[\frac{\partial R_{n}\left(r_{i}\right)}{\partial r_{i}}\right]\right\} \Psi_{n}\left(\mu_{i}, \phi_{i}\right) \\
& v_{n}\left(r_{j}, \mu_{j}, \phi_{j} \mid r_{i}, \mu_{i}, \phi_{i}\right)=\left\{f\left(\mu_{i}, \phi_{i}\right) R_{n}\left(r_{j}\right) \Psi_{n}\left(\mu_{j}, \phi_{j}\right)\right. \\
& \left.+g\left(\mu_{i}, \phi_{i}\right)\left[\frac{\partial R_{n}\left(r_{j}\right) \Psi_{n}\left(\mu_{j}, \phi_{j}\right)}{\partial r_{i}}\right]\right\} .
\end{aligned}
$$

For the case of a constant-potential surface,

$$
\begin{gathered}
\chi_{m}=R_{m}\left(r_{i}\right) \Psi_{m}\left(\mu_{i}, \phi_{i}\right) \\
v_{n}=R_{n}\left(r_{j}\right) \Psi_{n}\left(\mu_{j}, \phi_{j}\right) \\
F_{m n}= \begin{cases}2 \pi, & m=n \\
0, & m \neq n,\end{cases}
\end{gathered}
$$

where the coordinates $\left(r_{i}, \theta_{i}, \phi_{i}\right)$ and $\left(r_{j}, \theta_{j}, \phi_{j}\right)$ are defined in Fig. 8. We have

$$
\begin{aligned}
\mathbf{r}_{j}=\left[\begin{array}{l}
r_{j x} \\
r_{j y} \\
r_{j z}
\end{array}\right]_{O_{j}}=\mathbf{r}_{j i}+\mathbf{r}_{i}=\mathbf{C}_{o_{j}, O}\left\{\left[\begin{array}{l}
r_{j i, x} \\
r_{j i, y} \\
r_{j i, z}
\end{array}\right]_{o}\right. \\
\left.+\mathbf{C}_{O, O_{i}}\left[\begin{array}{c}
r_{i} \sin \left(\theta_{i}\right) \cos \left(\phi_{i}\right) \\
r_{i} \sin \left(\theta_{i}\right) \sin \left(\phi_{i}\right) \\
r_{i} \cos \left(\theta_{i}\right)
\end{array}\right]_{o_{i}}\right\} \\
r_{j}=\sqrt{r_{j x}^{2}+r_{j y}^{2}+r_{j z}^{2}} \\
\phi_{j}=\tan ^{-1}(y / x) \\
\theta_{j}=\cos ^{-1}\left(z / r_{j}\right) .
\end{aligned}
$$

Here $[\vdots]_{H}$ represents the position vector based on coordinate system $H, \mathbf{C}_{H, H^{\prime}}$ is the coordinate transform matrix from $H^{\prime}$ to $H . O_{j}, O_{i}$, and $O$ are the coordinate systems characterized by $\left(x_{j}, y_{j}, z_{j}\right),\left(x_{i}, y_{i}, z_{i}\right)$, and $(x, y, z)$, respectively. The $\mathbf{h}_{j}$ in [B5] is defined as

$$
\mathbf{h}_{j}=\left[\begin{array}{c}
h_{j, 1} \\
h_{j, 2} \\
\vdots \\
h_{j, N}
\end{array}\right],
$$

where

$h_{j, n}=\int_{0}^{2 \pi} \int_{-1}^{1} h_{i}\left(\mu_{i}, \phi_{i}\right) \Psi_{n}^{*}\left(\mu_{i}, \phi_{i}\right) d \mu_{i} d \phi_{i}$,

$$
n=1,2, \ldots, N \text {. }
$$

For the case of a constant-potential surface, [B12b] leads to

$$
\begin{aligned}
& h_{j, 1}=2 \pi \sqrt{2} \Psi_{\mathrm{S}} \\
& h_{j, n}=0, \quad n=2,3, \ldots, N .
\end{aligned}
$$

\section{ACKNOWLEDGMENT}

This work is supported by the National Science Council of the Republic of China under Grant NSC84-2214-E002-005. 


\section{REFERENCES}

1. Krozel, J. W., and Saville, D. A., J. Colloid Interface Sci. 150, 365 (1992).

2. Carnie, S. L., and Chan, D. Y. C., J. Colloid Interface Sci. 155, 297 (1993).

3. Carnie, S. L., Chan, D. Y. C., and Gunning, J. S., Langmuir 10, 2993 (1994).

4. Ninham, B. W., and Parsegian, V. A., J. Theor. Biol. 31, 405 (1971).

5. Prieve, D. C., and Ruckenstein, E., J. Theor. Biol. 56, 205 (1976).

6. Carnie, S. T., Chan, D. Y. C., and Stankovich, J., J. Colloid Interface Sci. 165, 116 (1994).

7. Carnie, S. T., and Chan, D. Y. C., J. Colloid Interface Sci. 161, 260 (1993).

8. Krozel, J. W., J. Colloid Interface Sci. 163, 437 (1994).

9. Kuin, A., J. Chem. Soc. Faraday Discuss. 90, 235 (1990).

10. Vreeker, R., Kuin, A. J., Den Boer, D. C., Hoekstra, L. L., and Agterof, W. G. M., J. Colloid Interface Sci. 145, 138 (1992).

11. Miklavic, S. J., Chan, D. Y. C., White, L. R., and Healy, T. W., J. Phys. Chem. 98, 9022 (1994).

12. Grant, M. L., and Saville, D. A., J. Colloid Interface Sci. 171, 35 (1995).

13. Wang, T. K., and Audebert, R., J. Colloid Interface Sci. 119, 459 (1987)
14. Glendinning, A. B., and Russel, W. B., J. Colloid Interface Sci. 93, 95 (1983).

15. Ohshima, H., Adv. Colloid Interface Sci. 53, 77 (1994).

16. Ohshima, H., J. Colloid Interface Sci. 162, 487 (1994).

17. Ohshima, H., J. Colloid Interface Sci. 170, 432 (1995).

18. Ohshima, H., J. Colloid Interface Sci. 168, 255 (1994).

19. Lyklema, J., "Fundamentals of Interface and Colloid Science.' Academic Press, London, 1991.

20. Hunter, R. J., “Foundations of Colloid Science,', Vol. I. Oxford Univ. Press, London, 1989.

21. Hildebrand, F. B., 'Method of Applied Mathematics.' Prentice-Hall, New York, 1954.

22. Spiegel, M. R., "Mathematic Handbook of Formulas and Tables." McGraw-Hill, New York, 1968.

23. Hsu, J. P., and Tseng, M. T., J. Chem. Phys. 104, 242 (1996).

24. Reddy, J. N., "An Introduction to the Finite Element Method.", McGraw-Hill, New York, 1984.

25. Gluckman, M. J., Pfeffer, R., and Weinbaum, S., J. Fluid Mech. 50, 705 (1971).

26. Hassonjee, Q., Ganatos, P., and Pfeffer, R., J. Fluid Mech. 197, 1 (1988).

27. Callier, F., and Desoer, C., ''Linear System Theory.' Springer-Verlag, Hong Kong, 1992. 